\title{
Rab5a-mediated autophagy regulates the phenotype and behavior of vascular smooth muscle cells
}

\author{
JIN-YUN TAN ${ }^{1 *}$, LUO-QI JIA ${ }^{2 *}$, WEI-HAO SHI ${ }^{1}$, QING HE ${ }^{1}$, LEI ZHU ${ }^{1}$ and BO YU ${ }^{3}$ \\ ${ }^{1}$ Department of Surgery, Huashan Hospital of Fudan University, Shanghai 200040; \\ ${ }^{2}$ Shanghai Key Laboratory of Female Reproductive Endocrine Related Diseases, \\ Obstetrics and Gynecology Hospital of Fudan University, Shanghai 200011; \\ ${ }^{3}$ Department of Surgery, Pudong Hospital of Fudan University, Shanghai 201399, P.R. China
}

Received October 9, 2015; Accepted August 23, 2016

DOI: $10.3892 / \mathrm{mmr} .2016 .5774$

\begin{abstract}
Rab5a, a key member of the Rab family of GTPases, was determined to be a regulator of vascular smooth muscle cell (VSMC) proliferation and migration. However, the exact regulatory mechanism remains unclear. As Rab5a has been shown to be associated with autophagy, which is essential for the conversion of VSMCs from a contractile to a synthetic phenotype in order to prevent cell death due to oxidative stress. The present study hypothesized that autophagy may be responsible for the proliferation and migration of VSMCs via the Rab5a protein. The aim of the present study was to evaluate the effect of Rab5a on autophagy in VSMCs. The human aorta vascular smooth muscle cell line, T/G HA-VSMCs, was treated with small interfering (si)RNA against Rab5a and/or platelet-derived growth factor (PDGF). Following treatment, the phenotype transition of the VSMCs was evaluated by detecting the mRNA and protien expression levels of VSMC molecular markers using reverse transcription-quantitative polymerase chain reaction and western blotting, respectively. In addition, autophagy in VSMCs was evaluated by western blotting for autophagy-associated proteins, flow cytometry of acidic vesicular organelles, punctate fluorescence of microtubule associated protein light chain 3 and transmission electron microscopy of typical scattered double-membrane vacuolar structures. Additionally, the proliferation, migration, cell cycle and apoptotic response of VSMCs were detected by sulforhodamine B assay, transwell assay and flow cytometry, respectively. The results revealed that transfection with siRNA against Rab5a led to a significant decrease in Rab5a protein
\end{abstract}

Correspondence to: Professor Bo Yu, Department of Surgery, Pudong Hospital of Fudan University, 2800 Gongwei Road, Pudong, Shanghai 201399, P.R. China

E-mail: paul.yubo@hotmail.com

*Contributed equally

Key words: Rab5a, vascular smooth muscle cells, autophagy, phenotype transition, ERK1/2 signaling pathway expression, while the reduced expression trend of Rab5a was rescued by intervention with PDGF. Furthermore, cells transfected with siRNA against Rab5a inhibited the autophagy of VSMCs. Downregulated Rab5a inhibited the phenotype transition of VSMCs. Additionally, downregulated Rab5a led to slowed cell growth, decreased numbers of migrated cells, decreased numbers of cells at the G0-G1 phase and a higher apoptosis rate. However, PDGF significantly rescued these phenomena caused by siRNA against Rab5a. These results indicated that Rab5a-mediated autophagy may regulate the phenotype transition and cell behavior of VSMCs through the activation of the extracellular-regulated kinase $1 / 2$ signaling pathway.

\section{Introduction}

Vascular smooth muscle cells (VSMCs), as the primary cellular components of the normal blood vessel wall, are associated with the integrity of vascular structure, regulation of vascular function and stability of vascular lesions (1). Notably, VSMCs are considered to be vital to the initiation and progression of atherosclerotic lesions (2). In response to trauma and altered hemodynamics of vascular reconstruction, the phenotypic modulation of VSMCs from a contractile to a synthetic phenotype is observed during atherogenesis, which promotes not only the migratory and proliferative capacity of VSMCs, but also the synthesis of extracellular matrix proteins $(3,4)$. Therefore, it is essential to understand the molecular mechanism underlying the phenotype transition and cell behavior of VSMCs.

Rab proteins are Ras-related small GTPases, which may regulate exocytic and endocytic membrane trafficking by vesicle docking and fusion $(5,6)$. As a key member of the Ras GTPase superfamily, Rab5a has been shown to be associated with not only the heterozygous fusion of early endosomes and endocytic vesicles, but also the homomorphous fusion between early endosomes (7). Ravikumar et al (8) suggested that Rab5a can promote autophagosome formation, indicating that Rab5a is associated with autophagy. In addition, Rab5a may influence the morphogenesis and metastasis of various cancer types, including breast cancer, cervical cancer, ovarian cancer and hepatocellular carcinoma (9-12). As the pathogenesis of 
intimal hyperplasia is somewhat similar to neoplasia, Rab5a may also be involved in the intimal hyperplasia and arterial restenosis. A previous study indicated that Rab5a is involved in VSMC proliferation and migration (13), while autophagy induced by platelet-derived growth factor (PDGF) serves an essential role in the conversion of VSMCs from the contractile to synthetic phenotype in order to prevent cell death due to oxidative stress (14). Therefore, the present study hypothesized that autophagy may be responsible for the proliferation and migration of VSMCs, and that Rab5a was essential in this process.

In the present study, a human aorta vasuclar smooth muscle cell line, referred to as T/G HA-VSMCs, was treated with small interfering (si)RNA against Rab5a and/or PDGF, and the phenotype transition and cell behaviors, including proliferation, cell cycle, migration, apoptosis and autophagy, were assessed. The present study aimed to reveal the effects of Rab5a on autophagy in VSMCs, and whether the phenotype transition and cell behaviors of VSMCs are accompanied by autophagy.

\section{Materials and methods}

Cell culture and treatment. T/G HA-VSMCs were obtained from American Type Culture Collection (Rockefeller, MD, USA). The cells were cultured in Dulbecco's modified Eagle's medium (DMEM; Gibco; Thermo Fisher Scientific, Inc., Waltham, MA, USA) containing $10 \%$ fetal bovine serum (FBS; Hyclone, Logan, UT, USA), penicillin (100 U/ml) and streptomycin $(100 \mathrm{mg} / \mathrm{ml})$ at $37^{\circ} \mathrm{C}$ with $5 \% \mathrm{CO}_{2}$. The cells were transfected with control siRNA (siC), Rab5a siRNA (siR; a pool of four siRNAs; Dharmacon Research, Lafayette, CO, USA), siC combined with PDGF ( $\mathrm{siC}+\mathrm{P} ; 20 \mathrm{ng} / \mathrm{ml}$; R\&D Biosystems, Minneapolis, MN, USA) and siR combined with PDGF (siR + P; $20 \mathrm{ng} / \mathrm{ml}$ ) prior to experiments. Transfection was performed using DharmaFECT transfection reagent in serum-free medium (GE Healthcare Life Sciences, Chalfont, UK) following manufacturer's protocol.

Reverse transcription-quantitative polymerase chain reaction $(R T-q P C R)$. Following treatment with siRNA and/or PDGF for $24 \mathrm{~h}$, the total RNA from cells was obtained using TRIzol reagent (Invitrogen; Thermo Fisher Scientific, Inc.), according to the manufacturer's protocol. The RNA (25 nM) was subsequently reverse transcribed using the RevertAid First-Strand cDNA Synthesis kit (Fermentas, Vilnius, Lithuania). PCR amplification was performed with the SYBR Green Premix Ex Taq kit (Takara Bio., Inc., Dalian, China). Primer sequences are listed in Table I. The PCR program included denaturation at $95^{\circ} \mathrm{C}$ for $10 \mathrm{sec}$, amplification with 40 cycles at $95^{\circ} \mathrm{C}$ for $5 \mathrm{sec}$ and $60^{\circ} \mathrm{C}$ for $31 \mathrm{sec}$, and a final $2 \mathrm{~min}$ extension at $72^{\circ} \mathrm{C}$. Finally, the $2^{-\Delta \Delta \mathrm{Cq}}$ method (15) was used to calculate the expression levels of genes, normalized against the levels of GAPDH.

Western blotting analysis. Following treatment for $48 \mathrm{~h}$ with siRNA and/or PDGF, the cells were lysed in radioimmunoprecipitation buffer (Beyotime Institute of Biotechnology, Inc., Jiangsu, China) containing $1 \mathrm{mM}$ phenylmethanesulfonyl fluoride. The supernatants were acquired by
Table I. Primer sequences.

\begin{tabular}{ll}
\hline Gene & \multicolumn{1}{c}{ Primer sequence $\left(5^{\prime} \rightarrow 3^{\prime}\right)$} \\
\hline $\begin{array}{l}\text { Rab5a } \\
\text { Forward } \\
\text { Reverse }\end{array}$ & CAGTTCAAACTAGTACTTCTGG \\
$\alpha-S M A$ & GCTAGGCTATGGTATCGTTCTTG \\
Forward & \\
Reverse & GCGTGGCTATTCCTTCGTTA \\
Calponin & ATGAAGGATGGCTGGAACAG \\
Forward & \\
Reverse & AGCTAAGAGAAGGGCGGAAC \\
Vimentin & CATCTGCAGGCTGACATTGA \\
Forward & \\
Reverse & AAAACACCCTGCAATCTTTCAGA \\
Osteopontin & CACTTTGCGTTCAAGGTCAAGAC \\
Forward & \\
Reverse & GGACAGCCGTGGGAAGG \\
GAPDH & TCAATCACATCGGAATGCTCA \\
Forward & GCACCGTCAAGGCTGAGAAC \\
Reverse & TGGTGAGACGCCAGTGGA
\end{tabular}

$\alpha$-SMA, $\alpha$-smooth muscle actin.

centrifugation at $13,440 \times \mathrm{g}$ for $15 \mathrm{~min}$ at $4^{\circ} \mathrm{C}$. The proteins (30 $\mu \mathrm{g} /$ lane) were separated and subsequently transferred onto a polyvinylidene difluoride membrane (EMD Millipore, Billerica, MA, USA). The membranes were blocked with 5\% non-fat milk at room temperature for $2 \mathrm{~h}$. Following blocking, the membranes were incubated with specific primary antibodies overnight at $4^{\circ} \mathrm{C}$ and then incubated with horserdish peroxidase-conjugated secondary antibodies (cat. nos. A0181; A0216; A0208; 1:1,000; Beyotime Institute of Biotechnology, Inc.) at room temperature for $2 \mathrm{~h}$. The antibodies used were as follows: anti-Rab5a (cat. no. 2143; 1:1,000), anti-survivin (cat. no. 2803), anti-caspase-3 (cat. no. 9662; 1:1,000), anti-Beclin1 (cat. no. 3738; 1:1,000), anti-Light chain (LC)3-I/II (cat. no. 12741; 1:1,000), anti-phosphorylated (p-) (cat. no. 4060; 1:1,000) and total (t-)protein kinase B (Akt) (cat. no. 4691; 1:1,000), anti-p-mammalian target of rapamycin (mTOR; cat. no 5536; 1:1,000) and anti-t-mTOR (cat. no. 2972; 1:1,000), and p-extracellular signal-regulated kinase (ERK cat. no. 4370; 1:1,000) and t-ERK (cat. no. 4695; 1:1,000; all from Cell Signaling Technology, Inc., Danvers, MA, USA. Anti- $\alpha$-smooth muscle actin (SMA; cat. no. SAB2500963; $0.5 \mu \mathrm{g} / \mathrm{ml}$ ) and anti-calponin (cat. no. C2687; 1:10,000) were obtained from Sigma-Aldrich (St. Louis, MO, USA). Anti-osteopontin (cat. no. sc-10591; 1:200) was purchased from Santa Cruz Biotechnology, Inc. (Dallas, TX, USA), and anti-GAPDH (cat. no. AF0006; 1:1,000) was purchased from Beyotime Institute of Biotechnology, Inc. Following antibody incubations, an enhanced chemiluminescence assay (PerkinElmer, Waltham, MA, USA) was used to detect the proteins. GAPDH was used as a loading control. 
Cell proliferation assay. The proliferation of VSMCs was detected using a sulforhodamine B (SRB) assay. A total of 3.0x $10^{3} \mathrm{~T} / \mathrm{G}$ HA-VSMCs were seeded into 96 -well plates. The next day, the cells were assigned into the following four groups: i) siC group, cells treated with control siRNA; ii) $\mathrm{siC}+\mathrm{P}$ group, cells treated with control siRNA and PDGF; iii) $\mathrm{siR}$ group, cell treated with Rab5a siRNA; and iv) $\mathrm{siR}+\mathrm{P}$ group, cells treated with Rab5a siRNA and PDGF. Following treatment for 24,48 and $72 \mathrm{~h}$, the cells were fixed in $30 \%$ (w/v) trichloroacetic acid and were subsequently stained with $0.4 \%$ SRB (Sigma-Aldrich) diluted with $1 \%$ acetic acid for $30 \mathrm{~min}$ at $25^{\circ} \mathrm{C}$. The excess SRB was rinsed off using $1 \%$ $(\mathrm{v} / \mathrm{v})$ acetic acid and the proteins were dissolved in $10 \mathrm{mM}$ Tris solution. Finally, a microplate reader (iMark; Bio-Rad, Hercules, CA, USA) was used to measure the value of SRB at a wavelength of $570 \mathrm{~nm}$.

Migration analysis. The migration of T/G HA-VSMCs was detected using a Transwell system with 24-well inserts and $8 \mu \mathrm{m}$ pores (Corning Costar, Lowell, MA, USA). Matrigel (30 $\mu \mathrm{l}$; BD Biosciences, San Jose, CA, USA) was used to coat the inserts and $600 \mu \mathrm{l}$ complete medium was added into the lower chamber. Following treatment for $48 \mathrm{~h}$, trypsin was used to detach the cells and the cells were resuspended in fresh medium $(0.2 \mathrm{ml})$ without FBS at a concentration of $1 \times 10^{4}$ cells These cell suspensions were seeded into the upper chamber. Following incubation for $24 \mathrm{~h}$, the cells in the upper chamber were removed with cotton swabs, while non-migrating cells were gently removed and rinsed out with phosphate-buffered saline (PBS) three times. Cells in the lower chamber were fixed with methanol at room temperature for $30 \mathrm{~min}$ and were subsequently stained using hematoxylin at room temperature for $5 \mathrm{~min}$. Migrating cells were counted in six randomly selected representative fields of each insert by light microscopy (BX51; Olympus, Tokyo, Japan).

Cell cycle and apoptosis analysis. Flow cytometry was performed to detect the cell cycle and apoptosis in each group. Following treatment for $48 \mathrm{~h}$, the cells were trypsinized, collected and fixed with pre-cooled $70 \%$ ethanol overnight at $4^{\circ} \mathrm{C}$. The cells were subsequently centrifuged at $1,120 \mathrm{x} g$ for $10 \mathrm{~min}$ at $4^{\circ} \mathrm{C}$ and the ethanol was washed off with PBS. The cells were trypsinized for $30 \mathrm{~min}$ at $37^{\circ} \mathrm{C}$. Propidium iodide (PI; $50 \mu \mathrm{g} / \mathrm{ml}$; Sigma-Aldrich) was used to stain the cells for $30 \mathrm{~min}$ at $25^{\circ} \mathrm{C}$ for cell cycle analysis.

Cell apoptosis analysis was performed using a Vybrant ${ }^{\circledR}$ Apoptosis assay kit (Invitrogen; Thermo Fisher Scientific, Inc.). Following treatment for $48 \mathrm{~h}$, the cells were co-stained with annexin V and PI, according to the manufacturer's protocol. The results were detected using flow cytometry (FACS Calibur; BD Biosciences, Franklin Lakes, NJ, USA). The data regarding cell cycle and apoptosis were acquired using CellQuest 7.0 software (Beckman Coulter, Brea, CA, USA) and subsequently analyzed using ModFit3.0 software (Verity Software House, Maine, ME, USA).

Acridine orange staining assay. To observe the characteristics of autophagic cell death, acidic vesicle organelles (AVOs) were stained with acridine orange (Sigma-Aldrich). Following treatment for $48 \mathrm{~h}$, the cells in different groups were stained
A

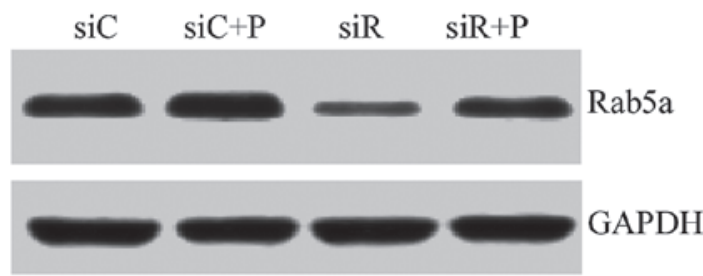

B

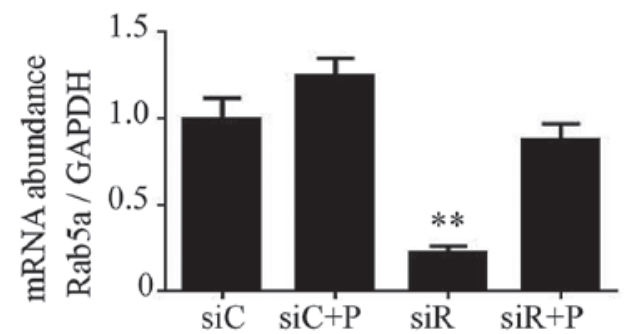

Figure 1. Expression of Rab5a in T/G HA-VSMCs treated in various manners. (A) The protein expression levels of Rab5a were determined by western blotting analysis following various treatments. GAPDH was used as a loading control. (B) The mRNA expression levels of Rab5a were determined by reverse transcription-quantitative polymerase chain reaction analysis following various treatments. The data were quantified and mRNA expression levels were determined relative to the expression of GAPDH. The data are presented as the mean \pm standard deviation $\left(^{* *} \mathrm{P}<0.01\right.$ compared with the siC group.) si, siRNA; C, control; R, Rab5a; P, PDGF; PDGF, platelet-derived growth factor; T/G HA-VSMC, human aorta-vascular smooth muscle cell line.

with acridine orange $(1 \mu \mathrm{g} / \mathrm{ml})$ for $15 \mathrm{~min}$ at $37^{\circ} \mathrm{C}$ in the dark. The cells were subsequently rinsed with PBS, collected with trypsin and then resuspended in PBS, supplemented with $1 \%$ FBS. Finally, the fluorescence emission was detected using CellQuest 7.0 software.

Detection of autophagy with LC3 localization. To further reveal the autophagy of cells in each group, the cells were co-transfected with pEGFP-LC3 (National Institute for Basic Biology, Okazaki, Japan) using Lipofectamine2000 (Invitrogen; Thermo Fisher Scientific, Inc.). Following transfection for $48 \mathrm{~h}$, the cells were fixed in $95 \%$ ethanol in the dark and fluorescence was detected by fluorescent microscopy (BX51; Olympus).

Transmission electron microscopy (TEM). TEM was performed to observe the formation of autophagosomes. Following treatment for $48 \mathrm{~h}$, the cells in each group were fixed in $3 \%$ glutaraldehyde at $25^{\circ} \mathrm{C}$ for $2 \mathrm{~h}$. The cells were subsequently fixed in $1 \%$ osmium tetroxide, and were sectioned and embedded in LX112 plastic. Finally, uranyl acetate combined with lead citrate was used to stain the ultrathin sections, and electron micrographs were obtained by TEM (Philips Medical Systems, BG Eindhoven, The Netherlands).

Statistical analysis. Statistical analysis was performed using SPSS version 15.0 software (SPSS Inc., Chicago, IL, USA). All data were expressed as the mean \pm standard deviation. Comparisons among different groups were analyzed by one-way analysis of variance, while comparisons between two groups were analyzed by Student's t-test. $\mathrm{P} \leq 0.05$ was considered to indicate a statistically significantly difference. 
A
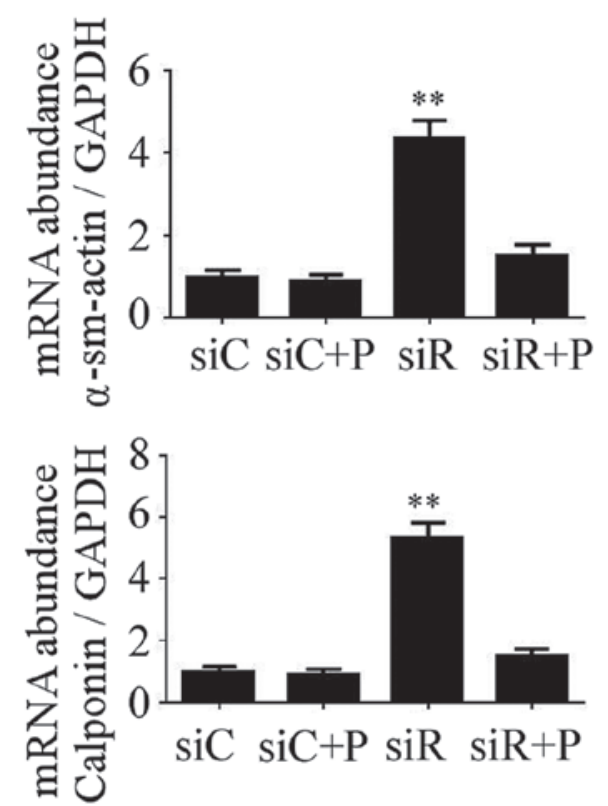
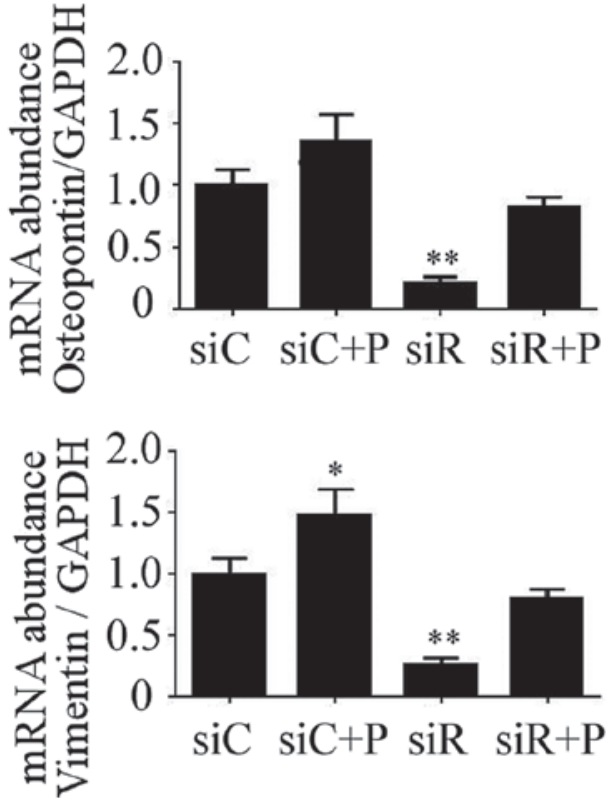

B

$\mathrm{siC} \quad \mathrm{SiC}+\mathrm{P} \quad \mathrm{siR} \quad \mathrm{siR}+\mathrm{P}$

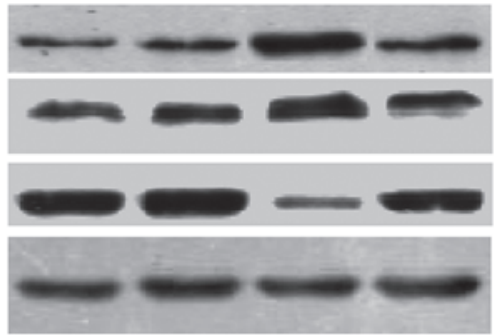

$\alpha-$ sm-actin

Calponin

Osteopontin

GAPDH

Figure 2. Expression of molecular markers of the VSMC phenotype following various treatments. (A) The mRNA expression levels of $\alpha$-sm-actin, Calponin, Osteopontin and Vimentin were determined by reverse transcription-quantitative polymerase chain reaction in T/G HA-VSMCs treated with siC, siR, siC $+\mathrm{P}$, and $\mathrm{siR}+\mathrm{P}$. The mRNA expression levels were normalized against that of GAPDH. The data are presented as the mean \pm standard deviation ("P $<0.05$ and ${ }^{* *} \mathrm{P}<0.01$ compared with the siC group.) (B) The protein expression levels of $\alpha$-sm-actin, Calponin and Osteopontin were determined by western blotting analysis. The protein and mRNA expression changes were confirmed to be the same. GAPDH was used as a loading control. si, siRNA; C, control; R, Rab5a; P, PDGF; PDGF, platelet-derived growth factor; T/G HA-VSMC, human aorta-vascular smooth muscle cell line; $\alpha$-sm-actin, $\alpha$-smooth muscle actin.

\section{Results}

Effect of Rab5a siRNA transfection and PDGF on the expression of Rab5a in T/G HA-VSMCs. The knockdown efficiency of Rab5a siRNA transfection and the effects of PDGF on T/G HA-VSMCs were determined using RT-qPCR and western blotting. As a result, transfection with siRNA against Rab5a led to a significant decrease in the mRNA and protein expression levels of Rab5a $(\mathrm{P}<0.01)$; however, the expression of Rab5a in the siR $+\mathrm{P}$ group was relatively close to the siC group (Fig. 1). These data revealed that Rab5a was effectively downregulated by Rab5a siRNA transfection and the reduced expression trend of Rab5a can be rescued by the intervention of PDGF in T/G HA-VSMCs.

Effect of Rab5a siRNA transfection and PDGF on the phenotype transition of T/G HA-VSMCs. The expression of molecular markers of the T/G HA-VSMC phenotype, including $\alpha$-SMA, calponin, osteopontin and vimentin, were detected to evaluate the phenotypic transition of T/G HA-VSMCs in different groups. As a result, the mRNA expression levels of $\alpha$-SMA and calponin were significantly increased, while osteopontin and vimentin were significantly reduced in the siR group compared with the siC group $(\mathrm{P}<0.01)$. However, the expression of these proteins was restored to normal levels by the intervention of PDGF, which was relatively close to siC (Fig. 2A). Similarly, the changes in the protein expression levels of $\alpha$-SMA, calponin and osteopontin were consistent with that of the mRNA level (Fig. 2B). These results indicated that Rab5a may promote the phenotypic transition of $\mathrm{T} / \mathrm{G}$ HA-VSMCs.

Effect of Rab5a siRNA transfection and PDGF on the proliferation, cell cycle and migration of T/G HA-VSMCs. In addition to the phenotypic transition of T/G HA-VSMCs, the proliferation, cell cycle and migration of T/G HA-VSMCs were investigated in the different groups. Compared with the siC group, the growth of the cells was significantly reduced in the siR group at $24(\mathrm{P}<0.05), 48(\mathrm{P}<0.01)$ and $72 \mathrm{~h}(\mathrm{P}<0.01)$. This growth trend was rescued by the intervention of PDGF in the $\mathrm{siR}+\mathrm{P}$ group (Fig. 3A). According to the cell cycle analysis, the percentage of cells in the siR group was significantly decreased in the $\mathrm{G} 0 / \mathrm{G} 1$ phase and increased at the $\mathrm{G} 2 / \mathrm{M}$ and $\mathrm{S}$ phases; however, the percentage of cells at the G0/G1, G2/M and S phases were 
A

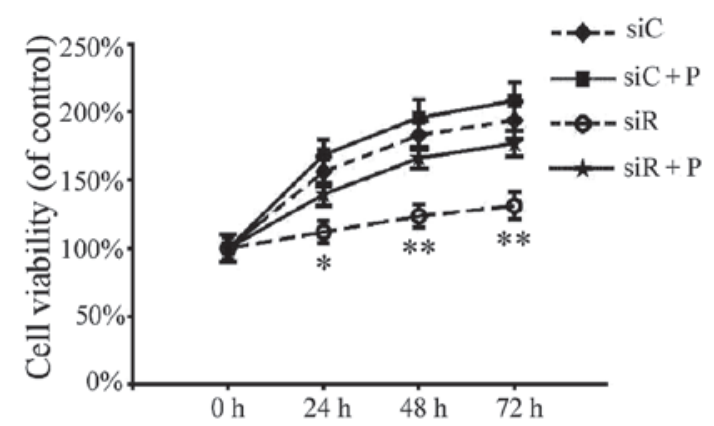

B
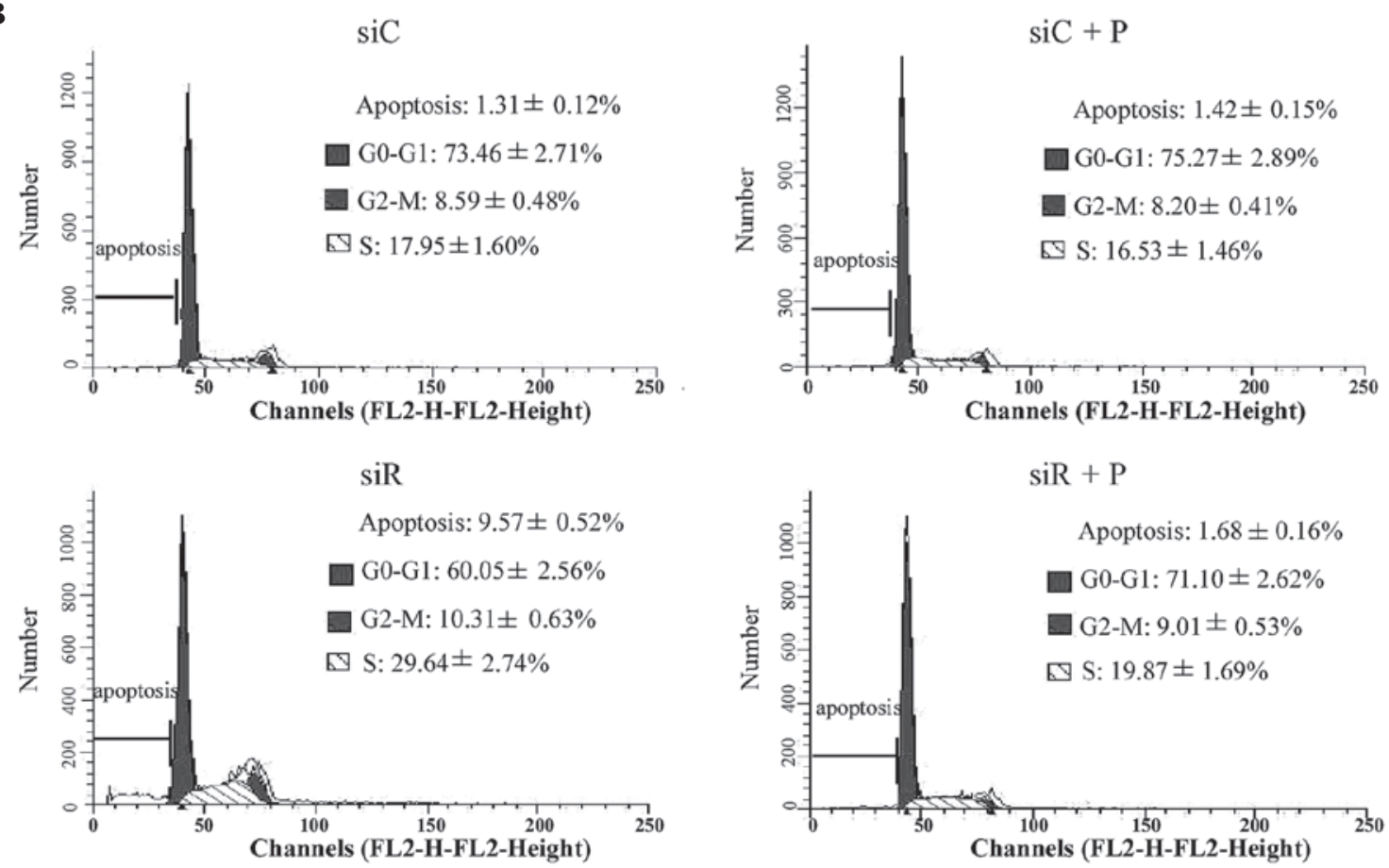

C
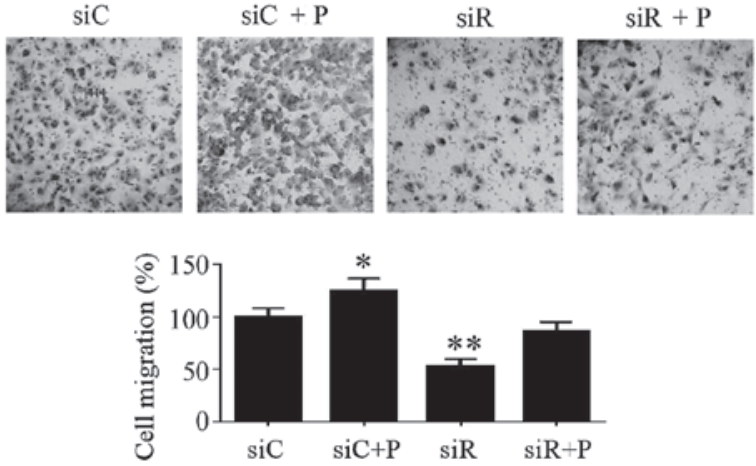

Figure 3. Proliferation, cell cycle and migration in T/G HA-VSMCs following various treatments. (A) A sulforhodamine B assay was used to demonstrate the growth of the cells following treatment that compared with $\mathrm{siC}$, siR, siC $+\mathrm{P}$ and $\mathrm{siR}+\mathrm{P}$ at 24,48 , and $72 \mathrm{~h}$,. (B) Cell cycle analysis was performed by flow cytometry following treatment with $\mathrm{siC}, \mathrm{siR}, \mathrm{siC}+\mathrm{P}$ and $\mathrm{siR}+\mathrm{P}$. The percentage of apoptotic, G0-G1, G2-M or S phase cells were calculated. (C) Microscopy observation (magnification, $\mathrm{x} 20$ ) of the cells was performed following migration assay. The percentage of cells migrating was calculated. Quantified data are presented as the mean \pm standard deviation ${ }^{(* *} \mathrm{P}<0.01$ and ${ }^{*} \mathrm{P}<0.05$ compared with the siC group.) si, siRNA; C, control; R, Rab5a; P, PDGF; PDGF, platelet-derived growth factor; T/G HA-VSMC, human aorta-vascular smooth muscle cell line.

not significantly changed in the $\mathrm{siR}+\mathrm{P}$ group compared with the siC group (Fig. 3B). These data indicated that Rab5a modulated T/G HA-VSMC proliferation at different phases of the cell cycle. In addition, compared with the $\mathrm{siC}$ group, the average number of migrated cells in the siR group was revealed to be significantly decreased $(\mathrm{P}<0.01)$. However, the percentage of migrated cells was not markedly changed in the si $\mathrm{R}+\mathrm{P}$ group. Additionally, more migrated cells were observed in the $\mathrm{siC}+\mathrm{P}$ compared with the $\mathrm{siC}$ group $(\mathrm{P}<0.05 ; \mathrm{Fig} .3 \mathrm{C})$. These results indicated that the knockdown of Rab5a can inhibit T/G HA-VSMCs migration and that PDGF treatment can reverse the decreased migration level in Rab5a siRNA transfected cells. 
A
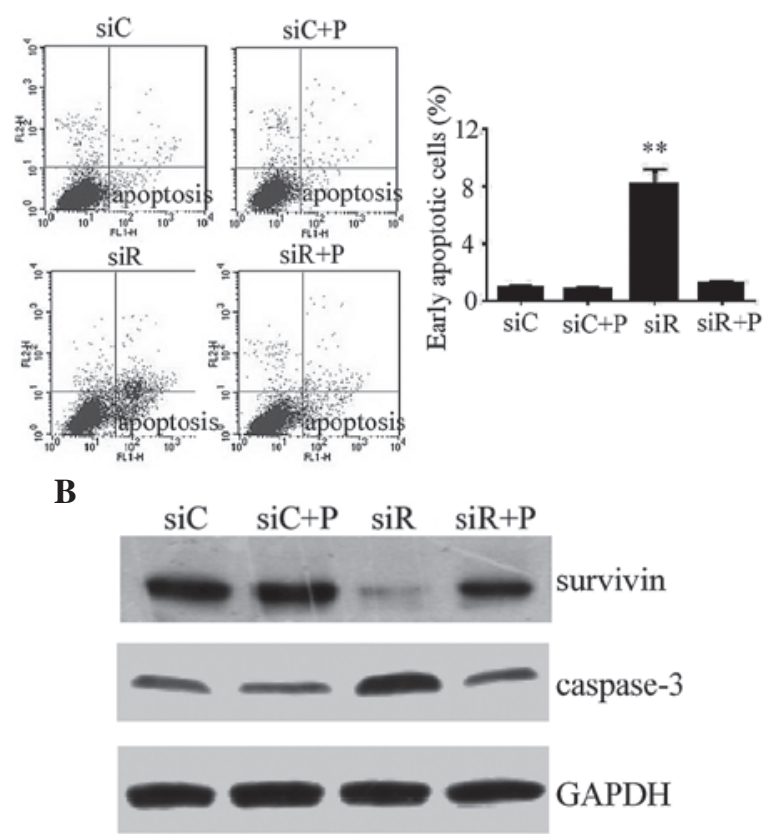

Figure 4. Apoptotic response in T/G HA-VSMCs following various treatments. (A) Flow cytometry was performed to detemin the percentage of cells undergoing apoptosis following treatment with $\mathrm{siC}, \mathrm{siR}, \mathrm{siC}+\mathrm{P}$ and $\mathrm{siR}+\mathrm{P}$. The data are presented as the mean \pm standard deviation $\left({ }^{* *} \mathrm{P}<0.01\right.$ compared with the siC group.) (B) The protein expression levels of apoptosis-associated proteins, survivin and caspase-3, were determined by western blotting analysis. GAPDH was used as a loading control. si, siRNA; C, control; R, Rab5a; P, PDGF; PDGF, platelet-derived growth factor; T/G HA-VSMC, human aorta-vascular smooth muscle cell line.

Effect of Rab5a siRNA transfection and PDGF on apoptosis in $T / G H A$-VSMCs. The early apoptotic rate was quantitatively analyzed by flow cytometry and the results demonstrated that cells transfected with siRNA against Rab5a exhibit a significantly higher apoptotic rate compared with the siC group $(\mathrm{P}<0.01)$, while the percentage of apoptotic cells was significantly reduced by the intervention of PDGF (Fig. 4A). In addition, the expression of the apoptotic proteins survivin and caspase- 3 was detected by western blotting. These results revealed reduced expression of survivin and increased expression of cleaved caspase- 3 in the siR group compared with the siC group, while a combination treatment with PDGF and siRNA (siCon or siRab) transfection did not alter the expression levels of survivin and caspase-3 (Fig. 4B).

Effect of Rab5a siRNA transfection and PDGF on autophagy in $T / G$ HA-VSMCs. The autophagy in T/G HA-VSMCs from each group was first detected by flow cytometry. As a result, the percentage of cells with AVOs were significantly decreased $(\mathrm{P}<0.01)$, while autophagy returned to a normal degree following treatment with PDGF (Fig. 5A). In addition, the autophagy in these cells was also analyzed by fluorescence localization of LC3. As a result, the percentage of cells with punctate fluorescence was significantly reduced in the siR group $(\mathrm{P}<0.05)$, while no difference was observed in the other three groups (Fig. 5B). Additionally, VSMCs were visualized by TEM to examine the formation of autophagosomes. It was revealed that Rab5a siRNA transfection caused a significantly reduced number of autophagosomes with typical scattered double-membrane vacuolar structures $(\mathrm{P}<0.05)$, while the number of phagosomes in cells treated with PDGF remained unchanged compared with the siC group (Fig. 5C). Furthermore, the expression levels of the autophagic proteins Beclin1, LC3-I and LC3-II were also analyzed by western blotting. As an autophagy-related protein, Beclin1 is required for the initiation of autophagy. Additionally, LC3-II formation is also a confirmed marker of autophagy. As the formation of LC3-II is transient and the protein can be rapidly degraded in the lysosome, a conversion of LC3-I to LC3-II may also indicate autophagy in cells. Compared with the siC group, the protein expression of Beclin1 and the conversion of LC3-I to LC3-II was significantly reduced in the siR group, whereas it was elevated to a normal level following treatment with PDGF (Fig. 5D).

Effect of Rab5a siRNA transfection and PDGF on the PI3K/AKT/mTOR and ERKI/2 signaling pathways in $T / G$ HA-VSMCs. To assess the mechanism of Rab5a siRNA transfection-related autophagy, the PI3K/AKT/mTOR and ERK1/2 signaling pathways in T/G HA-VSMCs were assessed by western blotting. The result revealed that compared with the siC group, the expression levels of p-AKT, p-mTOR and p-ERK were markedly reduced in the siR group, while PDGF recovered the normal expression of these proteins. In addition, no differences were observed in the expression levels of t-AKT, t-mTOR and t-ERK among these four groups (Fig. 6). The results suggested that the MAPK/ERK pathway may be involved in Rab5a-related autophagy, which did not occur via the inhibition of the classical PI3K/AKT/mTOR pathway.

\section{Discussion}

It is generally accepted that accelerated proliferation and migration of VSMCs are essential for the development of neointimal hyperplasia, which is also a key pathological feature of atherosclerosis and vascular reconstruction with restenosis (16). The present study revealed that downregulated expression of Rab5a inhibited the phenotypic transition, proliferation, migration and autophagy of T/G HA-VSMCs, while it increased apoptosis in T/G HA-VSMCs. However, PDGF treatment reversed these changes. Furthermore, Rab5a-related autophagy may be induced via the ERK pathway, however, not the PI3K/AKT/mTOR pathway.

Previous studies have demonstrated that phenotype transition in VSMCs is regulated by numerous factors, including contractile agonists and extracellular matrix components (17-19). Notably, the present study found that downregulation of Rab5a resulted in increased expression levels of $\alpha$-SMA and calponin, as well as decreased expression levels of osteopontin and vimentin. These results indicated that Rab5a may be associated with the phenotype transition from contractile to synthetic in VSMCs; however, the underlying mechanism remains unclear. Su et al (20) suggested that Rab5a was associated with autophagy induced by hepatitis $\mathrm{C}$ virus non-structural protein 4B, while autophagy was an essential regulator for the phenotype transition of VSMCs (14). Therefore, it was speculated that autophagy may serve a vital role in the effect of Rab5a on the phenotype transition of VSMCs. In addition, it was revealed that PDGF treatment 
A
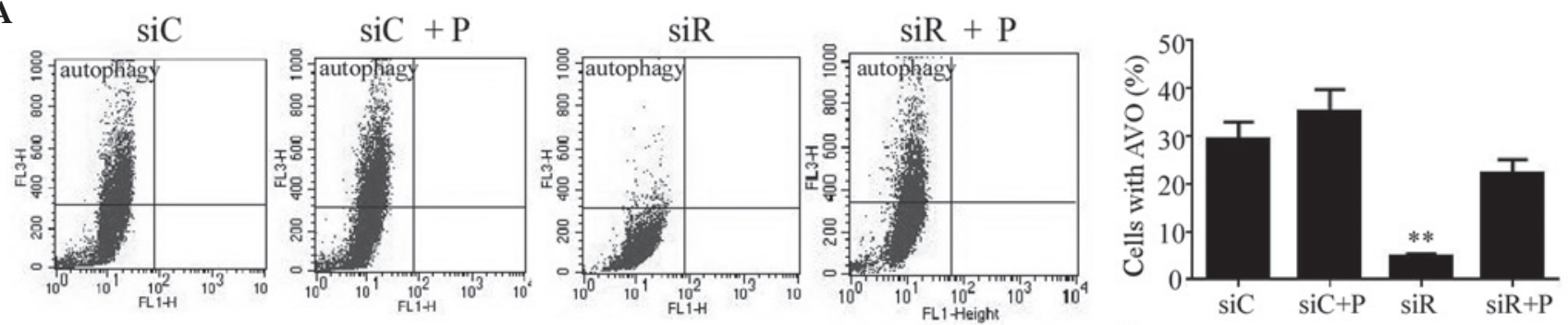

B
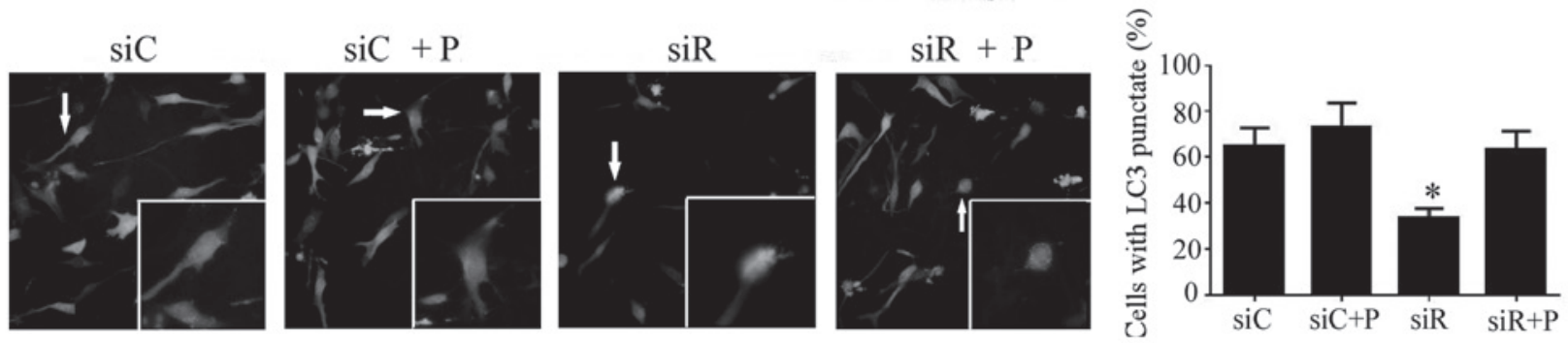

C

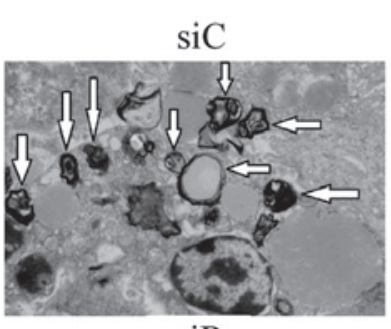

SiR
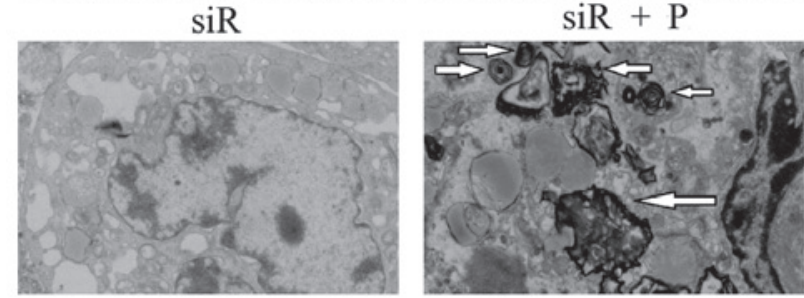

$\mathrm{SiC}+\mathrm{P}$

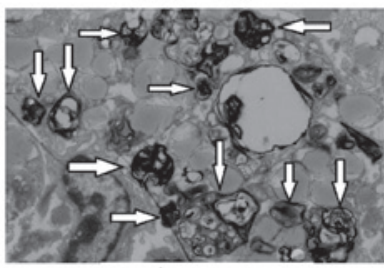

$\mathrm{siR}+\mathrm{P}$

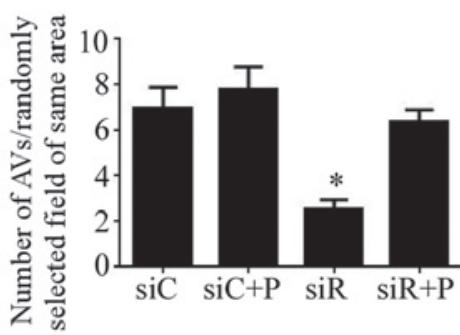

D

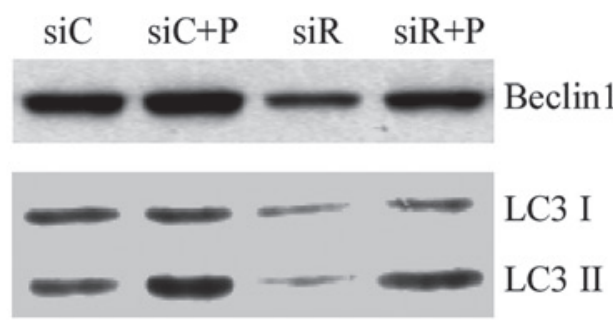

Figure 5. Autophagy in T/G HA-VSMCs following various treatments. (A) Flow cytometry was performed to determine the percentage of cells with AVOs as a sign of autophagy following treatment with $\mathrm{siC}, \mathrm{siR}, \mathrm{siC}+\mathrm{P}$ and siR $+\mathrm{P}$. (B) Representative microscopy images (magnification, $\mathrm{x} 40$ ) of fluorescence localization of LC3 were captured and the percentage of cells with punctate fluorescence was calculated. (C) Transmission electron microscopy (magnification, 1,000x) was performed to determine the number of autophagosomes with typical scattered double-membrane vacuolar structures (arrows). (D) The protein expression levels of Beclin1, LC3 I and LC3 II were determined by western blotting analysis. Quantified data are presented as the mean \pm standard deviation ${ }^{* *} \mathrm{P}<0.01$ and ${ }^{*} \mathrm{P}<0.05$ compared with the siC group.) AVOs, acidic vesicular organelles; LC3, light chain 3; si, siRNA; C, control; R, Rab5a; P, PDGF; PDGF, platelet-derived growth factor; T/G HA-VSMC, human aorta-vascular smooth muscle cell line.

can significantly reverse the phenotype switching of VSMCs caused by Rab5a siRNA transfection. Notably, PDGF is usually overexpressed in atherosclerotic lesions and can promote VSMC proliferation $(21,22)$, which may induce autophagy and phenotype switching in VSMCs (14). These results further indicated that the phenotypic transition of VSMCs induced by Rab5a may be regulated by autophagy.

Furthermore, the present study demonstrated that Rab5a promoted the proliferation and migration of T/G HA-VSMCs. Similarly, our previous study also suggested a critical role for
Rab5a in rat VSMC proliferation and migration (13). However, the exact mechanism remains to be elucidated. Autophagy was considered to be the basic catabolic mechanism that is essential for cell survival, differentiation, development and the cellular response to stress via the regulation of the actions of lysosomes (23). Du et al (24) suggested that autophagy can promote angiogenesis by AKT activation in aortic endothelial cells, while increased angiogenesis may promote tube formation and endothelial cell migration (25). In addition, in lung cancer cells, autophagy also facilitated cell proliferation, 


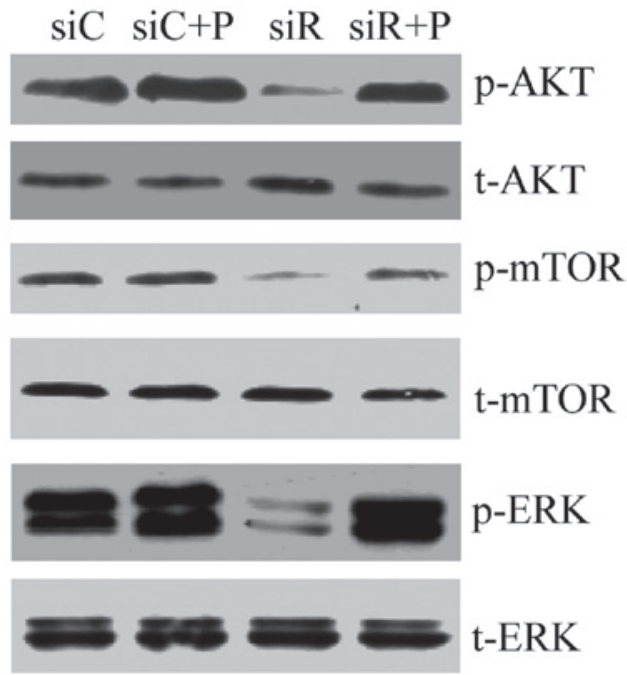

Figure 6. PI3K/AKT/mTOR and ERK1/2 signaling pathways in T/G HA-VSMCs following various treatments. The mRNA expression levels of p-AKT, t-AKT, p-mTOR, t-mTOR, p-ERK and t-ERK were determined by western blotting analysis following treatment with siC, siR, siC $+\mathrm{P}$ and siR + P. p-, phosphorylated; t-, total; Akt, protein kinase B; mTOR, mammalian target of rapamycin; ERK, extracellular signal-regulated kinase; si, siRNA; C, control; R, Rab5a; P, PDGF; PDGF, platelet-derived growth factor; T/G HA-VSMC, human aorta-vascular smooth muscle cell line.

migration and invasion by increasing the expression levels of various cytokines, including interleukin 6 , vascular endothelial growth factor A and matrix metallopeptidase 2 (26). Therefore, the present study speculated that the proliferation and migration of T/G HA-VSMCs induced by Rab5a may also be regulated by autophagy.

The crosstalk between apoptosis and autophagy is very complex. Autophagy is a physiological process accompanied by the degradation of disordered cellular components, while apoptosis is programmed cell death. Under stress circumstances, cells exhibit a stress adaptation by inducing low levels of autophagy, and the intrinsic and extrinsic pathways of apoptosis are inhibited in order to avoid cell death (27). However, in certain circumstances, autophagy acts as a key regulator to activate the programmed cell death pathway, causing massive cell death and eventually leading to functional impairment in vivo (28). In the present study, Rab5a was revealed to inhibit the apoptosis of VSMCs, which exhibited reduced expression of survivin, high expression levels of caspase-3 and a higher apoptosis rate. However, Rab5a was demonstrated to induce autophagy in VSMCs that exhibited significantly reduced expression of Beclin1, to downregulated the conversion of LC3-I to LC3-II, reduce AVOs, LC3 punctate fluorescence and autophagosomes with typical scattered double-membrane vacuolar structures in Rab5a siRNA transfected cells. This may be explained by the fact that autophagy confers cytoprotection to VSMCs. In addition, Shen et al (29) observed that high autophagy levels induced cell death in tumor cells receiving anticancer treatments, while low levels of autophagy may promote cell survival by removing disordered intracellular proteins and organelles (30). These two previous studies indicated that the cell survival or death was associated with the level of autophagy, which may also explain the present results. Therefore, Rab5a-induced autophagy may protect against the onset of apoptosis, and the inhibition of autophagy by Rab5a siRNA transfection can trigger the apoptosis of $\mathrm{T} / \mathrm{G}$ HA-VSMCs, and subsequently regulate the proliferation and migration of T/G HA-VSMCs.

The present study further examined various signaling pathways involved in autophagy. Previously, the PI3K/AKT/mTOR signaling pathway has been considered to be crucial in the regulation of cellular survival, differentiation, proliferation, metabolism, migration and angiogenesis (31). Additionally, it was also a well-known pathway in the regulation of autophagy in mammalian cells, which can activate autophagy by the inhibition of AKT and mTOR $(32,33)$. However, the present results demonstrated that the expression of phosphorylated AKT and mTOR was reduced by the knockdown of Rab5a, demonstrating that Rab5a-related autophagy was independent of the PI3K/AKT/mTOR pathway. Notably, a reduced expression trend of Rab5a was also observed, to be consistent with the phosphorylation of ERK1/2. The MAPK/ERK pathway served a primary role in the promotion of cellular proliferation in response to growth factors (34) and the induction of autophagy by the activation of the ERK1/2 pathway $(35,36)$. These results indicated that Rab5a-related autophagy correlated with the ERK1/2 pathway; however, not the PI3K/AKT/mTOR pathway.

Rab5a is pivotal for autophagy in VSMCs, and the phenotype transition, proliferation, cell cycle, migration and apoptosis induced by Rab5a may be regulated by autophagy via the activation of the ERK1/2 pathway. The present results supported a critical role for Rab5a in atherogenesis. However, further studies are required to detail the underlying molecular mechanisms of Rab5a-induced autophagy.

\section{Acknowledgements}

The authors would like to thank the Electron Microscopy Laboratory of Fudan University for technical assistance in the TEM analysis. This work was supported by the Outstanding Leaders Training Program of Pudong Health Bureau of Shanghai (PWRI2011-02), the Project of Science and Technology Commission of Shanghai Municipality (12jc1408101) and the Shanghai Pudong District Science Project (PKJ2012-Y68) .

\section{References}

1. Owens GK, Kumar MS and Wamhoff BR: Molecular regulation of vascular smooth muscle cell differentiation in development and disease. Physiol Rev 84: 767-801, 2004.

2. Pyle AL and Young PP: Atheromas feel the pressure: Biomechanical stress and atherosclerosis. Am J Pathol 177: 4-9, 2010.

3. Rudijanto A: The role of vascular smooth muscle cells on the pathogenesis of atherosclerosis. Acta Med Indones 39: 86-93, 2007.

4. Mack CP: Signaling mechanisms that regulate smooth muscle cell differentiation. Arterioscler Thromb Vasc Biol 31: 1495-1505, 2011.

5. Jordens I, Marsman M, Kuijl C and Neefjes J: Rab proteins, connecting transport and vesicle fusion. Traffic 6: 1070-1077, 2005.

6. Grosshans BL, Ortiz D and Novick P: Rabs and their effectors: Achieving specificity in membrane traffic. Proc Natl Acad Sci USA 103: 11821-11827, 2006

7. Hutagalung AH and Novick PJ: Role of Rab GTPases in membrane traffic and cell physiology. Physiol Rev 91: 119-149, 2011. 
8. Ravikumar B, Imarisio S, Sarkar S, O'Kane CJ and Rubinsztein DC: Rab5 modulates aggregation and toxicity of mutant huntingtin through macroautophagy in cell and fly models of Huntington disease. J Cell Sci 121: 1649-1660, 2008.

9. Yang PS, Yin PH, Tseng LM, Yang CH, Hsu CY, Lee MY, Horng CF and Chi CW: Rab5A is associated with axillary lymph node metastasis in breast cancer patients. Cancer Sci 102: 2172-2178, 2011.

10. Liu SS, Chen XM, Zheng HX, Shi SL and Li Y: Knockdown of Rab5a expression decreases cancer cell motility and invasion through integrin-mediated signaling pathway. J Biomed Sci 18 : $58,2011$.

11. Zhao Z, Liu XF, Wu HC, Zou SB, Wang JY, Ni PH, Chen XH and Fan QS: Rab5a overexpression promoting ovarian cancer cell proliferation may be associated with APPL1-related epidermal growth factor signaling pathway. Cancer Sci 101: 1454-1462, 2010.

12. Fukui K, Tamura S, Wada A, Kamada Y, Igura T, Kiso S and Hayashi N: Expression of Rab5a in hepatocellular carcinoma: Possible involvement in epidermal growth factor signaling. Hepatol Res 37: 957-965, 2007.

13. Ma Z, Wang H, Wu L, Zhui L, Shi W, Ma D, Chen Z and Yu B: RNAi-mediated Rab5a suppression inhibits proliferation and migration of vascular smooth muscle cells. Acta Cardiol 65 $507-514,2010$

14. Salabei JK, Cummins TD, Singh M, Jones SP, Bhatnagar A and Hill BG: PDGF-mediated autophagy regulates vascular smooth muscle cell phenotype and resistance to oxidative stress Biochem J 451: 375-388, 2013.

15. Livak KJ and Schmittgen TD: Analysis of relative gene expression data using real-time quantitative PCR and the $2^{-\Delta \Delta C T}$ method. Methods 25: 402-408, 2001.

16. Butoi E, Gan AM and Manduteanu I: Molecular and functional interactions among monocytes/macrophages and smooth muscle cells and their relevance for atherosclerosis. Crit Rev Eukaryot Gene Expr 24: 341-355, 2014.

17. Garat C, Van Putten V, Refaat ZA, Dessev C, Han SY and Nemenoff RA: Induction of smooth muscle alpha-actin in vascular smooth muscle cells by arginine vasopressin is mediated by c-Jun amino-terminal kinases and p38 mitogen-activated protein kinase. J Biol Chem 275: 22537-22543, 2000.

18. Hautmann MB, Thompson MM, Swartz EA, Olson EN and Owens GK: Angiotensin II-induced stimulation of smooth muscle alpha-actin expression by serum response factor and the homeodomain transcription factor MHox. Circ Res 81: 600-610, 1997.

19. Wang L, Zheng J, Du Y, Huang Y, Li J, Liu B, Liu CJ, Zhu Y, Gao Y, Xu Q, et al: Cartilage oligomeric matrix protein maintains the contractile phenotype of vascular smooth muscle cells by interacting with alpha(7)beta(1) integrin. Circ Res 106: 514-525, 2010.

20. Su WC, Chao TC, Huang YL, Weng SC, Jeng KS and Lai MM Rab5 and class III phosphoinositide 3-kinase Vps34 are involved in hepatitis C virus NS4B-induced autophagy. J Virol 85: 10561-10571, 2011

21. Myllärniemi M, Calderon L, Lemström K, Buchdunger E and Häyry P: Inhibition of platelet-derived growth factor receptor tyrosine kinase inhibits vascular smooth muscle cell migration and proliferation. FASEB J 11: 1119-1126, 1997.
22. Yu JC, Lokker NA, Hollenbach S, Apatira M, Li J, Betz A, Sedlock D, Oda S, Nomoto Y, Matsuno K, et al: Efficacy of the novel selective platelet-derived growth factor receptor antagonist CT52923 on cellular proliferation, migration, and suppression of neointima following vascular injury. J Pharmacol Exp Ther 298: 1172-1178, 2001

23. Levine B and Klionsky DJ: Development by self-digestion: Molecular mechanisms and biological functions of autophagy. Dev Cell 6: 463-477, 2004.

24. Du J, Teng RJ, Guan T, Eis A, Kaul S, Konduri GG and Shi Y: Role of autophagy in angiogenesis in aortic endothelial cells. Am J Physiol Cell Physiol 302: C383-C391, 2012.

25. Slevin M, Krupinski J, Rovira N, Turu M, Luque A, Baldellou M, Sanfeliu C, de Vera N and Badimon L: Identification of pro-angiogenic markers in blood vessels from stroked-affected brain tissue using laser-capture microdissection. BMC Genomics 10: 113, 2009.

26. Zhan Z, Xie X, Cao H, Zhou X, Zhang XD, Fan H and Liu Z: Autophagy facilitates TLR4-and TLR3-triggered migration and invasion of lung cancer cells through the promotion of TRAF6 ubiquitination. Autophagy 10: 257-268, 2014.

27. Lum JJ, DeBerardinis RJ and Thompson CB: Autophagy in metazoans: Cell survival in the land of plenty. Nat Rev Mol Cell Biol 6: 439-448, 2005

28. Platini F, Pérez-Tomás R, Ambrosio $\mathrm{S}$ and Tessitore L: Understanding autophagy in cell death control. Curr Pharm Des 16: 101-113, 2010

29. Shen S, Kepp O, Michaud M, Martins I, Minoux H, Métivier D, Maiuri MC, Kroemer RT and Kroemer G: Association and dissociation of autophagy, apoptosis and necrosis by systematic chemical study. Oncogene 30: 4544-4556, 2011.

30. Amaravadi RK: Autophagy-induced tumor dormancy in ovarian cancer. J Clin Invest 118: 3837-3840, 2008.

31. Hsieh AC, Liu Y, Edlind MP, Ingolia NT, Janes MR, Sher A, Shi EY, Stumpf CR, Christensen C, Bonham MJ, et al: The translational landscape of mTOR signalling steers cancer initiation and metastasis. Nature 485: 55-61, 2012.

32. Saiki S, Sasazawa Y, Imamichi Y, Kawajiri S, Fujimaki T, Tanida I, Kobayashi H, Sato F, Sato S, Ishikawa K, et al: Caffeine induces apoptosis by enhancement of autophagy via PI3K/Akt/mTOR/p70S6K inhibition. Autophagy 7: 176-187, 2011.

33. Wang RC, Wei Y, An Z, Zou Z, Xiao G, Bhagat G, White M, Reichelt $\mathrm{J}$ and Levine B: Akt-mediated regulation of autophagy and tumorigenesis through Beclin 1 phosphorylation. Science 338: 956-959, 2012

34. Shaul YD and Seger R: The MEK/ERK cascade: From signaling specificity to diverse functions. Biochim Biophys Acta 1773: 1213-1226, 2007.

35. Wang J, Whiteman MW, Lian H, Wang G, Singh A, Huang D and Denmark T: A non-canonical MEK/ERK signaling pathway regulates autophagy via regulating Beclin 1. J Biol Chem 284: 21412-21424, 2009 .

36. Cagnol S and Chambard JC: ERK and cell death: Mechanisms of ERK-induced cell death-apoptosis, autophagy and senescence. FEBS J 277: 2-21, 2010 . 